\title{
Formación profesional y la práctica supervisada en el Servicio Social brasileño
}

\section{Reginaldo Ghiraldelli}

Asistente Social. Doctor en Servicio Social

Universidad de Brasilia. Brasilia, Brasil

https://orcid.org/0000-0002-9229-7686 • rghiraldelli@unb.br

\begin{abstract}
Resumen
El artículo, basado en una reflexión teórica y también a partir de la experiencia académica con la supervisión de prácticas, tiene como objetivo presentar aportes sobre la formación en Servicio Social en Brasil. Hace énfasis en la práctica supervisada, en un escenario brasileño marcado por una profunda crisis política y económica, que provoca serios ataques, amenazas y recortes presupuestarios en la enseñanza superior pública y, al mismo tiempo, mediante medidas neoliberales alienta la proliferación de la enseñanza privada y a distancia. Este contexto, caracterizado por el recrudecimiento del conservadurismo, representa desafíos al Servicio Social, en lo que se refiere a la defensa de un proyecto de formación de calidad, crítico, laico y público en Brasil. Entre las formas y estrategias de lucha y resistencia en el ámbito de la profesión, se construyó colectivamente, durante la gestión de la ABEPSS (2009-2010), la Política Nacional de Práctica (PNE), con el objetivo de asegurar una dirección social crítica en el proceso de formación, teniendo como base las Directrices Curriculares de 1996, lo que ha exigido de la profesión esfuerzos cotidianos ante las adversidades coyunturales de la realidad brasileña.
\end{abstract}

Palabras clave: Servicio Social; Formación Profesional; Práctica Supervisada.

Recibido: 22/02/2019 | Aprobado: 15/10/2019

Esta obra está bajo una Licencia Creative Commons Atribución-NoComercial-CompartirIgual 4.0 Internacional.

¿Cómo citar este artículo? / How to quote this article?

Ghiraldelli, R. (2020). Formación Profesional y la Práctica Supervisada en el Servicio Social brasileño. Prospectiva. Revista de Trabajo Social e intervención social, (29), 39-56. doi: 10.25100/prts.v0i29.7554. 


\title{
Professional Training and Supervised Practice in the Field of Social Work in Brazil
}

\begin{abstract}
The article, based on a theoretical reflection and also on academic experience with the supervision of practices, aims to contribute to the aspect of training in the field of Social Work in Brazil. Emphasis is made on supervised practice in a context marked by a deep political and economic crisis, that provokes serious attacks, threats and budget cuts in public higher education and, at the same time, through neoliberal measures, encourages the proliferation of private and distance education. This context, characterized by the resurgence of conservatism, gives rise to challenges to Social Work, in terms of the defense of a high quality, critical, secular and public training project in Brazil. Among the forms and strategies of struggle and resistance in the field of the profession, the National Policy of Practice (PNE) was collectively constructed, during the management of ABEPSS (2009-2010), with the aim of ensuring critical social direction in the training process. The policy was based on the Curricular Guidelines of ABEPSS of 1996, and its execution has demanded constant effort from the profession in the face of the conjunctural adversities of the Brazilian reality.
\end{abstract}

Keywords: Social Work; Professional Training; Supervised Practice.

Sumario: 1. Introducción, 2. Formación profesional, práctica supervisada y la Política Nacional de Práctica, 3. La supervisión de las prácticas: concepción, perspectivas y tensiones, 4. La práctica supervisada curricular no obligatoria, 5. Las competencias de las Instituciones de Enseñanza, 6. Posibilidades y desafíos de la supervisión, 7. Apuntes sobre la supervisión a partir de las experiencias académicas vivenciadas, 8. La mercantilización de la educación ante la ofensiva neoliberal: retos para la formación profesional de calidad, 9. Conclusiones, 10. Referencias bibliográficas. 


\section{Introducción}

En los últimos años, se observa en Brasil un crecimiento de estudios, investigaciones y análisis sobre la formación y calificación profesional con énfasis en la práctica supervisada en Servicio Social. La supervisión se entiende como un contenido formativo de capacitación que se basa en los fundamentos teóricos-metodológicos-éticos y en las experiencias de los/las profesionales resultantes de la relación entre instituciones de enseñanza y espacios ocupacionales de trabajo de los/las asistentes sociales. El proceso de supervisión de prácticas ocurre simultáneamente en las instituciones de enseñanza y se llama supervisión académica y en los espacios laborales de los/las asistentes sociales, llamada supervisión de campo.

La supervisión de prácticas se adentró en la pauta de discusiones del Servicio Social brasileño principalmente a partir de la década de 1990, con la revisión curricular propuesta por la Asociación Brasileña de Enseñanza e Investigación en el Servicio Social (ABEPSS) para los cursos de graduación, en un escenario de profundas transformaciones societarias, que repercutieron en los espacios laborales, en las políticas sociales, en la relación Estadosociedad, en el escenario educativo, y consecuentemente en la formación y en el trabajo profesional de asistentes sociales.

La categoría profesional en Brasil, organizada a través del Consejo Federal de Servicio Social (CFESS), Consejo Regional de Servicio Social (CRESS), Asociación Brasileña de Enseñanza e Investigación en el Servicio Social (ABEPSS), Ejecutiva Nacional de Estudiantes de Servicio Social (ENESSO) y las Instituciones de Enseñanza ha pautado y problematizado cuestiones relativas a la práctica supervisada con vistas a defender y asegurar un proyecto de formación basado en la teoría social crítica y en consonancia con los principios del proyecto ético-político, que se afirma y se sostiene alineado a un proyecto societario que tiene como horizonte la igualdad, la justicia, la democracia, la libertad y la emancipación humana. La lucha de la categoría profesional también se orienta en torno a la defensa de una educación pública, de calidad, laica y de una formación profesional que contemple competencias teóricas, metodológicas, éticas, políticas y técnicas de los asistentes sociales. También se defiende la ampliación de concursos públicos, jornada de trabajo compatible con las conquistas de asistentes sociales brasileños ${ }^{1}$, además de condiciones de trabajo que no infrinjan prerrogativas éticas y técnicas, como por ejemplo el secreto y la autonomía profesional $^{2}$.

Innumerables son las acciones del Servicio Social en Brasil para asegurar una formación crítica, pública, de calidad, relacionada permanentemente con el ejercicio profesional y consonante con los principios del proyecto ético-político.

${ }^{1}$ Ley 12.317 de 2010 que establece la jornada de 30 horas de trabajo semanal para asistentes sociales. La aprobación de esta ley es el resultado de muchas luchas y organización de la categoría profesional en Brasil.

${ }^{2}$ De acuerdo con el Código de Ética Profesional de los Asistentes Sociales de Brasil.

Prospectiva. Revista de Trabajo Social e intervención social • No. $29 \bullet$ ene.jun. $2020 \bullet$ pp. 39-56 e-ISSN: 2389-993X • Doi: 10.25100/prts.v0i29.7554

Universidad del Valle - Cali, Colombia 
Por eso, no es posible pensar la formación desvinculada del trabajo profesional y descontextualizada de la realidad social. Para analizar la formación y el trabajo profesional, es imprescindible comprender las cuestiones estructurales y coyunturales del tiempo presente, pues el Servicio Social se sitúa en ese suelo histórico y las transformaciones societarias (económicas, políticas, culturales) inciden directamente en el conjunto de las profesiones (Netto, 1996), tanto en el aspecto formativo y de calificación, como en el trabajo. Siendo así, no se puede analizar el Servicio Social cerrado en sí mismo, de manera endógena (Iamamoto, 2007). Así, hay que comprender la profesión situada históricamente.

De este modo, comprender la formación profesional en Brasil en la contemporaneidad exige contextualizar la política educativa brasileña, que en las últimas décadas ha sido afectada por las orientaciones neoliberales. En este caso, las Instituciones de Enseñanza no quedaron exentas de ese proceso, pues, como forma de atender las prerrogativas del capital, la universidad se metamorfoseó, asumiendo un carácter operacional (Chauí, 2003), orientada a metas de productividad, rentabilidad, eficiencia y eficacia, distanciándose de su función y compromiso precoz, que es el de la producción de conocimientos orientados a las necesidades humanas y sociales de la sociedad.

También es importante considerar que en esta coyuntura, marcada por la reestructuración capitalista con prerrogativa neoliberal, que flexibiliza y precariza las relaciones de trabajo, son identificados fuertes ataques a los derechos sociales, como es el caso de las ofensivas (contra) reformas que se han realizado en Brasil desde los años 1990 y que representa innumerables retrocesos y regresión en el ámbito de los derechos de la población.

Los cambios en curso apuntan a la minimización de las responsabilidades del Estado con los servicios sociales, las políticas públicas y los derechos de la población. Y con ese lastre, la universidad pasa por un profundo proceso de mercantilización y precarización de las relaciones del trabajo, incidiendo en el ámbito de la enseñanza y de la investigación. Siendo así, la práctica supervisada, componente curricular obligatorio en el proceso de formación y calificación profesional, no está exenta de esos rebotes, lo que exige estrategias, alternativas y salidas colectivas en el enfrentamiento de esa realidad. En ese contexto de tantas adversidades, la categoría profesional de asistentes sociales brasileños, colectivamente organizados, elaboran la Política Nacional de Práctica Supervisada (PNE) en la gestión de la ABEPSS en el bienio 2009-2010.

Ese artículo, basado en una reflexión teórica-bibliográfica y también considerando la experiencia docente en la coordinación y en la supervisión de prácticas (en el ámbito académico), presenta, en líneas generales, la construcción de la Política Nacional de Práctica Supervisada en el Servicio Social brasileño, con sus principios y directrices, y los desafíos de su implementación ante el actual escenario neoliberal de mercantilización de la educación.

Prospectiva. Revista de Trabajo Social e intervención social • No. $29 \bullet$ ene.jun. $2020 \bullet$ pp. 39-56 e-ISSN: 2389-993X • Doi: 10.25100/prts.v0i29.7554

Universidad del Valle - Cali, Colombia 


\section{Formación profesional, práctica supervisada y la Política Nacional de Práctica}

En su artículo, Campanini (2010) describe la formación en Servicio Social en el contexto europeo destacando sus desafíos actuales, especialmente después del Acuerdo de Bolonia, que altera significativamente la lógica del sistema educativo.

Según Campanini (2010), la formación profesional y las prácticas supervisadas se abordan de manera diferente en Europa, dependiendo de las particularidades locales y regionales de cada país. También presenta las tendencias recientes en Europa con respecto a las políticas sociales y las relaciones pedagógicas, lo que tiene implicaciones directas para la formación profesional y la estructura curricular de los cursos de Servicio Social. Al presentar un "Estado del Arte" del Servicio Social en la realidad europea, señala similitudes y diferencias en la formación profesional entre los diversos países que conforman el continente europeo. Un ejemplo es que en algunos países prevalece la formación en instituciones de enseñanza privadas y en otros en instituciones públicas. El tiempo de formación también cambia de país a país, ya que en algunos el curso de Servicio Social se desarrolla en 3 años, en otros es de 4 años y en algunos casos de 5 años.

En cuanto a los contenidos y las disciplinas que subyacen a la formación, se encuentran aquellos vinculados a las ciencias humanas como la sociología, la pedagogía, la psicología, así como las asignaturas de política social y estudios jurídicos. También hay una diferenciación en la estructura del plan de estudios con respecto a los contenidos teóricos y prácticos, que implica la práctica supervisada. A partir de la investigación y los datos obtenidos, se observa la figura del supervisor, tutor, maestro o instructor de campo en el proceso de supervisión. Son nombres diferentes para designar el rol de supervisor (Campanini, 2010).

También, según Campanini (2010), se han implementado muchas medidas en los últimos años con respecto a la internacionalización, lo que contribuye al intercambio de experiencias de diferentes realidades, pero aún quedan muchas dificultades, como la movilidad de los estudiantes frente a los altos costos económicos y las habilidades lingüísticas. En este sentido, el desafío se plantea en el campo de la capacitación con respecto a la dimensión internacional e intercultural que proporciona intercambios y experiencias basadas en intercambios que incluyen profesores y estudiantes. Entre los esfuerzos presentados por las medidas adoptadas después del Acuerdo de Bolonia están la necesidad de garantizar mejoras y calidad en la educación, así como fortalecer la investigación. Además, pensar en una formación que no se centre solo en los intereses y necesidades del mercado.

\footnotetext{
Prospectiva. Revista de Trabajo Social e intervención social • No. $29 \bullet$ ene.jun. $2020 \bullet$ pp. 39-56 e-ISSN: 2389-993X • Doi: 10.25100/prts.v0i29.7554

Universidad del Valle - Cali, Colombia
} 
Campanini (2010) presenta otros desafíos presentes en la realidad europea, como el envejecimiento de la población, el intenso proceso de migración, la crisis, el desempleo y el aumento de la pobreza. Aún en el conjunto de desafíos para la formación en Servicio Social, destaca la necesidad y la importancia de incorporar debates sobre cuestiones culturales, étnicas, raciales y de género. La formación profesional, desde una perspectiva intercultural y el intercambio de experiencias de diferentes realidades, hace posible romper con el etnocentrismo. Además de identificar las desigualdades presentes en la realidad, es importante comprender los procesos de discriminación. Por esta razón, la formación profesional también debe incluir enfoques desde la perspectiva de los derechos humanos y la justicia social. Esto requiere pensar en la formación en Servicio Social desde una perspectiva internacional y cruzar los límites globales. Argumenta sobre la necesidad de comprender la dimensión política de la profesión y la conexión necesaria entre lo local y lo global. Por esto, según Campanini (2010), la formación debe permitir la dimensión crítica y reflexiva, combinando teoría y práctica para desarrollar habilidades y competencias para la práctica profesional.

Así, en Brasil, la supervisión de práctica, comprendida como parte esencial de la formación y calificación en Servicio Social, es una actividad curricular obligatoria que capacita pedagógicamente estudiantes para el trabajo profesional, siendo de competencia de las Instituciones de Enseñanza asegurar la inserción de estudiantes en campos de práctica, según lo preconizado por los proyectos pedagógicos de los cursos y las orientaciones de las Directrices Curriculares Nacionales (ABEPSS, 1996a).

Para la realización de la práctica supervisada se requiere supervisión continua y sistemática de asistentes sociales insertos en los espacios laborales, denominados/as supervisores/as de campo, y de docentes, también responsables por el acompañamiento de estudiantes en las actividades de práctica, denominados/as supervisores/as académicos/as.

El/la supervisor/a de campo y el/la supervisor/a académico/a son profesionales indispensables en el proceso de formación de estudiantes, pero es posible observar que, según Baptista-Lewgoy (2009), es necesario calificar el trabajo de articulación entre el/la supervisor/a de campo y el académico/a, así como de éstos con los demás docentes. Baptista-Lewgoy (2009) plantea que el seguimiento permanente y sistemático de la práctica supervisada exige de la supervisión un repensar continuo y sistemático de este proceso pedagógico que compone la formación profesional sin perder de vista la realidad social y las demandas presentadas para la profesión. 
Además de los/las supervisores/as de campo, académicos/as y los/las estudiantes, considera también importante compartir ese proceso de la supervisión con la Coordinación de Práctica, que, de acuerdo con la Política Nacional de Práctica (PNE), y entre sus innumerables atribuciones, acompaña de forma sistemática y continuada la práctica supervisada desde la selección, acreditación y apertura de vacantes por medio del contacto institucional con los campos de actuación profesional, propone, con la participación de los sujetos involucrados en el proceso, directrices para operacionalización de la política de prácticas en consonancia con las prerrogativas de la formación profesional, establece contactos institucionales con el objetivo de acompañar, evaluar y planificar actividades conjuntas entre Universidad (Instituciones de Enseñanza) y campo de práctica, propone la documentación necesaria para la realización de la práctica, produce y publicita material pedagógico relativo a la práctica, comparte las experiencias de la práctica, presenta los campos de prácticas, etc. Con el objetivo de evitar centralización y propiciar la participación democrática y colectiva, la PNE sugiere que la coordinación de prácticas constituya una Comisión de Práctica, formada por los sujetos involucrados, es decir, supervisores/as de campo, supervisores/as académicos/as y estudiantes, para que puedan acompañar y evaluar permanentemente el proceso de supervisión.

Otra cuestión que merece destacarse es que la supervisión de las prácticas de trabajo social, según lo establecido en el artículo 5 de la Ley de Reglamentación de la Profesión, Ley No. 8.662 de 1993, es una asignación privativa del/de la asistente social, no cabiendo a los/las profesionales de otras áreas acompañar, en calidad de supervisores/as, los/las estudiantes en prácticas de Servicio Social.

En el marco de las Directrices Curriculares (ABEPSS, 1996a) y en la PNE, la práctica supervisada no puede concebirse como mera ejecución de tareas y fuerza de trabajo barata para atender las necesidades del mercado e instituciones de empleo, sino como proceso pedagógico y formativo, que se constituye en la relación enseñanza-aprendizaje.

En este sentido, las Directrices Curriculares (ABEPSS, 1996a) explicitan los principios fundamentales de la formación profesional, la relación indisociable entre teoría y realidad, entre la dimensión investigativa e intervencionista. Por eso, pensar en la supervisión de prácticas es pensar en la relación teoría-realidad y en la investigación e intervención.

Además, la práctica supervisada no puede ser concebida de forma fragmentada y desvinculada de los demás componentes y contenidos curriculares en el proceso de formación. Debe estar vinculada y relacionada con los demás contenidos curriculares y disciplinas, con el objetivo de dialogar con los núcleos propuestos en las Directrices Curriculares, o sea, con el núcleo de los fundamentos teórico-metodológicos de la vida social, de la formación socio-histórica brasileña y los fundamentos del trabajo profesional (ABEPSS, 1996b). La práctica supervisada debe también conjugar las dimensiones que envuelven el proceso formativo, que son: dimensión teórico-metodológica, ético-política y técnico-operativa (ABEPSS, 1996b).

Prospectiva. Revista de Trabajo Social e intervención social • No. $29 \bullet$ ene.-jun. $2020 \bullet$ pp. 39-56 e-ISSN: 2389-993X • Doi: 10.25100/prts.v0i29.7554

Universidad del Valle - Cali, Colombia 
Según Baptista-Lewgoy (2009), la supervisión de prácticas es un espacio privilegiado de interlocución entre Instituciones de Enseñanza y el mundo del trabajo, colocando a los profesionales involucrados en este proceso la tarea de comprender la realidad inmediata e ir más allá de ella.

\section{La supervisión de las prácticas: concepción, perspectivas y tensiones}

Según Puig-Cruells (2015), no existe una única definición del término supervisión, por lo que puede hablarse de una actividad plural. Para ella, existen diferentes concepciones de supervisión la cual puede presentarse como formación, como un instrumento de control o como un lugar para la libre expresión de los/las profesionales y estudiantes. También destaca en su libro que el término supervisión proviene etimológicamente de las palabras latinas super (sobre) y videre (ver), que unidas significan 'visión desde arriba'. Si se analiza el vocablo supervisión independientemente de su contexto, éste remite a la acción de ver de forma ampliada.

De acuerdo con Puig-Cruells (2015), la supervisión es una práctica multiforme y se dirige a estudiantes, a personas en formación, a profesionales en ejercicio o a equipos que trabajan en una institución. Los profesionales que la ejercen, o sea, los/las supervisores, también proceden de distintos campos de conocimiento, y esta diversidad se acentúa por las numerosas referencias teóricas en las que se fundamenta su práctica. Esto permite realizar aproximaciones de diverso signo al fenómeno de la supervisión, tanto en cuanto a su concepción como a las diferentes condiciones de ejercerla.

Basado en las reflexiones presentadas por Puig-Cruells (2015), considero que la supervisión en Servicio Social provee a los estudiantes y asistentes sociales de un espacio de reflexión sobre la práctica, que se sitúa en el interfaz entre el aprendizaje, la formación, la educación y la contribución institucional. La supervisión permite potenciar el pensamiento, las capacidades y competencias profesionales. También favorece el aprendizaje, impide el acomodo acrítico a las situaciones, o sea, persigue interrogarse, actuar de forma reflexiva y contribuye a la generación de conocimiento. Sin embargo, la supervisión consiste en un espacio privilegiado para pensar y reflexionar críticamente, ya que el Servicio Social trabaja en situaciones altamente complejas. Por esto, la supervisión, según Puig-Cruells (2015), permite profundizar en las condiciones de su trabajo y su contribución a la reflexividad, a la ética y a la calidad de los servicios prestados a la población. Así mismo, se estudian y analizan, diferentes abordajes teóricos y prácticos de la supervisión, con el objetivo de esclarecer su práctica, y abrir el campo de investigación y profesionalización.

En el caso brasileño, además de la concepción contenida en las Directrices Curriculares (ABEPSS, 1996a), que prevé la práctica supervisada como actividad formativa y pedagógica que requiere supervisión continua y sistemática por un/a profesional en el campo institucional y por un/a profesor/a, la práctica supervisada también presenta saltos cualitativos en el ámbito legislativo-normativo a partir de la Ley de Pasantías en Brasil (Ley No. 11.788 de 2008) que asegura la práctica como actividad educativa y formativa. A partir de esto, se rompe con una cultura presente en la sociedad de incorporar a los/las estudiantes

Prospectiva. Revista de Trabajo Social e intervención social • No. $29 \bullet$ ene.-jun. $2020 \bullet$ pp. 39-56 e-ISSN: 2389-993X • Doi: 10.25100/prts.v0i29.7554

Universidad del Valle - Cali, Colombia 
en las instituciones como trabajo barato y/o en sustitución de profesionales, pues, según Oliveira (2009), la práctica supervisada, en muchos casos, asume el carácter de empleo y fuente de renta para los/las estudiantes, sobre todo en un contexto de crecimiento de las desigualdades sociales, de profundización de la pauperización e índices elevados de desempleo.

Así, de acuerdo con Vasconcelos (2009), además de vender su fuerza de trabajo por bajo precio, los/las estudiantes en prácticas tienden a evaluar esta experiencia únicamente por el aspecto de ser económicamente ventajoso y relevante para el currículo, fragilizando y distorsionando la concepción formativa de la práctica supervisada. Por eso, según Baptista-Lewgoy (2009), la práctica supervisada no es un lugar donde el/la estudiante ejerce el papel de sustituto del profesional, sino un espacio en el que reafirma su formación, no en una condición de empleado/a, sino de estudiante en práctica.

En este contexto, a pesar de los límites presentes en la legislación, es importante reconocer en esa coyuntura una conquista considerable en el plano jurídico-político sobre la concepción de prácticas, incluso ante las dificultades y obstáculos en su efectividad en Brasil.

Ramos (2009) plantea que también es importante reconocer avances en el Servicio Social en la contemporaneidad, resultante de la organización política y resistencia colectiva de la categoría y que se expresan en las Directrices Curriculares de la ABEPSS de 1996, en el Código de Ética Profesional de los Asistentes Sociales de 1993, en la Ley de Reglamentación de la Profesión, Ley No. 8.662 de 1993, en la Resolución CFESS No. 533 de 2008 (que regula la supervisión directa de prácticas) y en la Política Nacional de Prácticas (PNE), ABEPSS (2009).

La PNE, resultado de la participación colectiva de la categoría profesional, tiene su marco normativo, ético, político y teórico amparado en las Directrices Curriculares (ABEPSS, 1996a) y basado en la defensa de una práctica supervisada vinculada a un proyecto de formación crítico, comprometido con una sociedad democrática y emancipada.

Pero, incluso con avances y logros en el ámbito de la práctica supervisada, tanto en su concepción, como en su operacionalización, todavía se observan dilemas presentes y desafíos puestos a la profesión para asegurar las prerrogativas del proyecto ético-político, teniendo en cuenta que éste es un proyecto que camina en la contracorriente de los valores de la sociabilidad capitalista, al defender la libertad, la igualdad, la justicia, la democracia, la socialización de la riqueza producida y la emancipación humana.

Por eso, entre los innumerables desafíos de materialización de la PNE, Ramos (2009) destaca la necesidad de socializar el documento de la PNE con las instituciones de enseñanza y discutir con los profesionales la formación, la calificación y la construcción colectiva de estrategias para la materialización de los parámetros y directrices propuestos en el documento, o sea, convertirlo efectivamente en un instrumento cotidiano de resistencia a la lógica de la precarización de la enseñanza y del trabajo, afirmándolo en la dirección de la defensa de la calidad de la práctica supervisada, de la formación y de la calificación profesional.

Prospectiva. Revista de Trabajo Social e intervención social • No. $29 \bullet$ ene.jun. $2020 \bullet$ pp. 39-56 e-ISSN: 2389-993X • Doi: 10.25100/prts.v0i29.7554

Universidad del Valle - Cali, Colombia 


\section{La práctica supervisada curricular no obligatoria}

Con la PNE, otras cuestiones también emergieron como desafíos a ser enfrentados por la categoría profesional, como es el caso de la práctica supervisada curricular no obligatoria, pues, tanto la práctica supervisada curricular obligatoria como la práctica no obligatoria tienen como exigencia el acompañamiento permanente y sistemático en el proceso de supervisión, sea la supervisión académica, sea la supervisión de campo.

Según Vasconcelos (2009), la práctica supervisada no obligatoria, durante largo período, se conoció, equivocadamente, como una actividad extracurricular. Esta idea de extracurricular hacía que la práctica se comprenda como una actividad desvinculada del currículo y distanciada de la formación profesional, como si tuviera una finalidad diferente de la educativa y formativa, dispensando supervisión académica y de campo.

Delante de la mercantilización de la enseñanza y de la precarización que asola el mundo del trabajo, incluso los espacios ocupacionales que incorporan asistentes sociales, que también son parte de la clase trabajadora asalariada, innumerables son los desafíos para que las Instituciones de Enseñanza aseguren el acompañamiento pedagógico en la supervisión de prácticas a los/las estudiantes, tanto en lo que se refiere a la práctica supervisada obligatoria, como la no obligatoria.

Además, desde la elaboración de la PNE, las gestiones de la ABEPSS han emprendido esfuerzos y realizado varias articulaciones con el objetivo de reglamentar la PNE ante el Consejo Nacional de Educación (CNE), lo que sería también un importante logro para la categoría profesional en el sentido de asegurar una directriz nacional, para que las Instituciones de Enseñanza de todo el país implementen los principios orientadores de la política, pues, conforme a las orientaciones éticas, políticas y normativas de la profesión, corresponde a las instituciones de enseñanza asegurar condiciones para la realización de una práctica supervisada de calidad, además de la captación de vacantes y encaminamiento de estudiantes a los campos de prácticas. Las instituciones de enseñanza establecen el período de la práctica supervisada, que se distribuye en semestres, con la respectiva carga horaria, y definen en su proyecto pedagógico la existencia o no de la práctica no obligatoria, teniendo en cuenta que esta actividad es opcional y concebida como complementariedad en el proceso formativo.

\section{Las competencias de las Instituciones de Enseñanza}

Corresponde a las Instituciones de Enseñanza, en el momento de elaboración y/o revisión de los proyectos pedagógicos, evaluar requisitos indispensables para el ingreso de estudiantes en la práctica supervisada. En el caso, de establecer la conclusión de disciplinas fundamentales para inserción discente en el espacio ocupacional de trabajo de los/las asistentes sociales, como, por ejemplo, Ética Profesional, Cuestión Social, Fundamentos históricos, teóricos y metodológicos del Servicio Social, Política Social, entre otros contenidos curriculares.

Prospectiva. Revista de Trabajo Social e intervención social • No. $29 \bullet$ ene.-jun. $2020 \bullet$ pp. 39-56 e-ISSN: 2389-993X • Doi: 10.25100/prts.v0i29.7554

Universidad del Valle - Cali, Colombia 
Para dar materialidad a la PNE, las instituciones de enseñanza necesitan asegurar: coordinación de prácticas, una política de prácticas en consonancia con las leyes y las regulaciones actuales, docentes supervisores/as académicos/as para el seguimiento sistemático del proceso de supervisión, la realización de visitas al lugar de prácticas, reuniones, supervisiones individuales, en grupo y colectivas, cursos para la formación continuada, foros de supervisión como espacios estratégicos de fortalecimiento y resistencia de la categoría, y demás actividades que contribuyan a la calidad de ese proceso formativo. También deben asegurar los criterios establecidos en la PNE, como el máximo de 15 estudiantes tutelados/das por docente-supervisor/a académico/a. La relación de 1 estudiante por supervisor/a de campo por cada 10 horas semanales de trabajo del profesional, conforme preconiza la Resolución No. 533 de 2008 del CFESS, corresponde a los Consejos Regionales el seguimiento y fiscalización. Por eso, para que los Consejos puedan hacer ese seguimiento, corresponde a las Instituciones de Enseñanza el encaminamiento semestral de las planillas conteniendo las informaciones del/de la estudiante, del/de la supervisor/a académico/a, del/de la supervisor/a de campo y de la Institución donde se realizan las prácticas.

Además de estas exigencias, otro punto relevante se refiere a la documentación en el proceso de supervisión de la práctica, como: 1) plan de prácticas, entendido como planificación de las actividades y acciones que serán desarrolladas, y elaborado conjuntamente por los/las supervisores/as académico/a, de campo y estudiante, 2) las evaluaciones periódicas, como parámetro pedagógico del proceso, 3) el diario de campo, como instrumento cotidiano de registro crítico-analítico de las acciones, 4) los informes, como un recurso de análisis crítico de la realidad social y de las particularidades de la práctica, y 5) los proyectos de investigación/intervención, como insumos estimuladores de la capacidad creativa, crítica y propositiva de estudiantes, oriundos de las demandas y necesidades identificadas en el proceso de supervisión.

La documentación, como instrumento técnico de registro y sistematización del trabajo profesional y del proceso de supervisión, que proporciona subsidios para analizar críticamente la realidad social, no es un mero recurso burocrático, o un simple acto de obtención de datos y registro de informaciones. Corresponde, según Marconsin (2010), a uno de los momentos reflexivos de producción del conocimiento en que los/las estudiantes en prácticas relacionan e interpretan hechos y fenómenos vividos en la inmediatez de lo cotidiano.

En este sentido, planificar, documentar y evaluar constantemente las acciones profesionales prevé la aprehensión crítica de la realidad social, contribuyendo al conocimiento de medios y fines en las actividades y posicionamiento ético-político sobre la intencionalidad de la actuación.

Prospectiva. Revista de Trabajo Social e intervención social • No. $29 \bullet$ ene.jun. $2020 \bullet$ pp. 39-56 e-ISSN: 2389-993X • Doi: 10.25100/prts.v0i29.7554

Universidad del Valle - Cali, Colombia 
Es importante resaltar también la importancia de la socialización de experiencias de la práctica supervisada, como, por ejemplo, la implementación de los proyectos de investigación/intervención desarrollados por estudiantes y supervisores/as. En muchas situaciones, los/las estudiantes desarrollan sus proyectos de trabajo de conclusión de curso a partir de las experiencias vividas durante la práctica, lo que significa el estímulo a la producción de conocimiento y la realización de investigaciones a partir de la inserción en la práctica supervisada.

\section{Posibilidades y desafíos de la supervisión}

La práctica supervisada, como momento de la formación y calificación profesional, debe ser comprendida en el contexto social, impregnado de contradicciones presentes en la sociedad y, por eso, la supervisión de prácticas no puede estar subordinada a los determinantes mercantiles y adecuarse a patrones de adiestramiento, entrenamiento, restringido a acciones técnicas burocráticas, mecánicas y rutinarias. La práctica supervisada, entendida en su procesualidad, requiere postura investigativa, rigurosa y crítica de la realidad. La supervisión de prácticas extrapola la dimensión del aprendizaje a hacer, y reducirla, según Baptista-Lewgoy (2009) a la operacionalización de instrumentos y técnicas es sustraer del proceso de aprendizaje la apropiación de los procesos de trabajo del Servicio Social en su amplitud. La supervisión prevé conexión entre dimensión teórica, ética, política y técnica, de forma que posibilite la crítica a la inmediatez, al fragmento, al sentido común, a la naturalización de las desigualdades sociales y la banalización de la vida social.

Al analizar y contextualizar el Servicio Social en el marco de las transformaciones societarias en que las alteraciones en el mundo del trabajo y en las políticas educativas repercuten directamente en la formación y en el ejercicio profesional, se observa una precarización en las relaciones de trabajo que incide en los espacios ocupacionales, trayendo consecuencias para la captación de vacantes, pues hay profesionales que optan por no recibir estudiantes, alegando que el ambiente de actuación es precario, no garantizando las condiciones éticas y técnicas adecuadas, como, por ejemplo, la inexistencia de sala privada que garantice el secreto y de espacio físico que acoge al estudiante. Sin embargo, en algunos casos observados, al recibir estudiantes, el/la asistente social adquiere mayor reconocimiento en los espacios institucionales, teniendo en cuenta las visitas realizadas en la supervisión, la participación en los foros, el acompañamiento pedagógico sistemático, la aproximación con la universidad y con la categoría por medio de las entidades representativas. Por otro lado, según Baptista-Lewgoy (2009), para muchos/as supervisores/as, el/la estudiante es entendido/a como 'oxígeno para la práctica', 'una mirada crítica', que marca la diferencia en lo cotidiano, pues exige que organicen mejor su intervención.

\footnotetext{
Prospectiva. Revista de Trabajo Social e intervención social • No. $29 \bullet$ ene.-jun. $2020 \bullet$ pp. 39-56 e-ISSN: 2389-993X • Doi: 10.25100/prts.v0i29.7554

Universidad del Valle - Cali, Colombia
} 


\section{Apuntes sobre la supervisión a partir de las experiencias académicas vivenciadas}

Ante las experiencias vividas y compartidas en los últimos años, sobre todo a partir de la inserción en la coordinación y la supervisión docente (académica) de las prácticas, se identifican innumerables retos en relación al proceso de supervisión de prácticas y la materialización de los principios y directrices de la PNE. Entre estos desafíos, se destacan: 1) la necesidad de creación y fortalecimiento de los foros de práctica, en todos los ámbitos (local, estatal, regional y nacional), como espacio de interlocución, articulaciones, resistencia y lucha colectiva de asistentes sociales, 2) las Instituciones de Enseñanza necesitan asegurar la práctica como contenido/disciplina continua, pues en algunos casos la supervisión académica no ocurre de forma sistemática, con encuentros semanales, sino esporádicos, lo que contribuye a debilitar este proceso, 3) necesidad de consolidación y reglamentación de la PNE, con el objetivo de asegurar un proyecto de formación profesional comprometido con la calidad y dirección social crítica, 4) la importancia de la interlocución entre universidades/instituciones de enseñanza y campos de prácticas, incluso con profesionales que aún no son supervisores/as de prácticas, 5) seguimiento sistemático y continuado de los campos de prácticas por el/la supervisor/a académico/a y por la coordinación de prácticas, 6) la defensa de la formación continuada, teniendo en cuenta que no se cierra en la graduación, 7) la consideración de que la práctica curricular no obligatoria debe seguir las mismas directrices de la práctica supervisada obligatoria, según lo preconizado en la Ley No. 11.788 de 2008 y en la PNE. La práctica no obligatoria debe tener acompañamiento didáctico-pedagógico, así como la práctica obligatoria. Se establece a criterio de la Institución de Enseñanza insertar o no la práctica no obligatoria en el proyecto pedagógico del curso, pues es opcional. Pero a partir de su incorporación en el proyecto pedagógico, la práctica no obligatoria debe seguir las mismas orientaciones y recomendaciones, pudiendo presentar diferenciaciones en la carga horaria establecida, 8) el desafío de no sucumbir a la perspectiva mercadológica de la práctica, volcada hacia intereses de la reproducción ampliada del capital, pues, en muchos casos, ocurren selecciones de práctica contemplando a estudiantes que ingresaron recientemente en los cursos, desconsiderando la práctica como actividad formativa pautada en el proceso de enseñanza y aprendizaje. Esto descalifica la concepción de práctica supervisada, que queda bajo los dictámenes del mercado, pues estudiantes recién ingresados en la universidad aún no adquirieron los conocimientos teóricos básicos y habilidades para su inserción en campo de práctica.

La práctica supervisada no puede asumir la forma de empleo y fuente de renta. Además, en la actualidad, se observa el crecimiento del perfil de estudiantes trabajadores/as, lo que requiere discusiones sobre las políticas de asistencia estudiantil que garanticen la permanencia de esos/as estudiantes en los cursos.

Prospectiva. Revista de Trabajo Social e intervención social • No. $29 \bullet$ ene.-jun. 2020 • pp. 39-56 e-ISSN: 2389-993X • Doi: 10.25100/prts.v0i29.7554

Universidad del Valle - Cali, Colombia 


\section{La mercantilización de la educación ante la ofensiva neoliberal: retos para la formación profesional de calidad}

Con el proceso de mercantilización de la educación y de expansión de los cursos de Servicio Social en Brasil, se identifica una ampliación de la demanda por vacantes de prácticas, teniendo en cuenta el crecimiento exponencial de la enseñanza superior privada ${ }^{3}$ (presencial y a distancia ${ }^{4}$ ) y pública (a través del REUNI ${ }^{5}$ ), que buscan convenios de práctica. Con la interiorización de las universidades y de los cursos, esto se vuelve más agravante, pues municipios de pequeño y mediano tamaño no poseen, en muchos casos, el número suficiente de profesionales (asistentes sociales) para supervisar la práctica. Esto provoca, muchas veces, el desplazamiento de estudiantes a municipios vecinos para la realización de la práctica. En estos casos, ante la falta de compromiso del Estado con la educación brasileña, universidades públicas, con bajos (o incluso ausentes) recursos presupuestarios, tienen dificultades para asegurar las condiciones necesarias en la realización de la práctica. También hay dificultades para garantizar, en algunas instituciones de enseñanza, la relación de 1 docente-supervisor/a académico/a para 15 estudiantes debido al número restringido de profesores, teniendo en cuenta la precarización que alcanza el trabajo docente, intensificando y sobrecargando las actividades de esos/as trabajadores/as, además de la ausencia de la realización de concursos públicos para la ampliación del cuadro docente.

Así, es preciso analizar críticamente esta coyuntura para no caer en el riesgo de distorsionar el debate y culpabilizar y/o satanizar las normativas, resoluciones y políticas construidas y conquistadas arduamente por la categoría profesional sobre la base de un debate democrático y colectivo. Las conquistas profesionales en el ámbito políticonormativo representan la defensa de un proyecto de formación de calidad, basado en la teoría social crítica y de condiciones de trabajo que aseguran la autonomía, la ética, el respeto y la dignidad de los trabajadores asalariados, en el caso, asistentes sociales.

Por eso, es preciso defender la dimensión pedagógica y formativa de la práctica supervisada, garantizando calidad y condiciones necesarias para una formación crítica, sintonizada con los principios del proyecto ético-político. En este caso, se refuerza la importancia de las entidades representativas, como ABEPSS, CFESS/CRESS y ENESSO.

${ }^{3}$ En Brasil, de acuerdo con los datos del Ministerio de Educación, existían en el año 1995, un total de 894 Instituciones de Enseñanza Superior, siendo que 684 eran privadas. Ya en 2013, el número total saltó a 2.391 Instituciones, siendo que 2.090 eran privadas. En 2013, había un total de 328 Instituciones de Enseñanza Superior que ofrecían el curso de Servicio Social, siendo 62 Instituciones Públicas, 248 Instituciones Privadas (curso presencial) y 18 que ofrecían el curso en la modalidad a distancia. Se observa que prevalece en Brasil la formación de asistentes sociales en Instituciones Privadas (Fuente: MEC/INEP).

${ }^{4}$ El Decreto No. 5.622 de 2005, crea la Universidad Abierta de Brasil (UAB), instituyendo la educación a distancia $(\mathrm{EaD})$.

${ }^{5}$ REUNI: Plan de Reestructuración y Expansión de las Universidades Federales brasileñas, instituido por el Decreto No. 6.096 de 2007, durante el Gobierno de Luiz Inacio Lula da Silva (PT).

Prospectiva. Revista de Trabajo Social e intervención social • No. $29 \bullet$ ene.-jun. $2020 \bullet$ pp. 39-56 e-ISSN: 2389-993X • Doi: 10.25100/prts.v0i29.7554

Universidad del Valle - Cali, Colombia 
Además, algunas peticiones y exigencias están puestas en la actualidad, como el aumento de demanda de prácticas supervisadas a los fines de semana, teniendo en cuenta el perfil de estudiantes trabajadores. Esto exige de las Instituciones de Enseñanza la necesidad de ampliación de los convenios y vacantes de prácticas que contemple esos segmentos, pero no hay un cuadro significativo de asistentes sociales que trabajen los fines de semana para atender la demanda. Esto se acentúa en las ciudades de pequeño y mediano tamaño, frente a un número reducido de profesionales. Otra cuestión presentada en la PNE y que ha sido espacio de amplio debate colectivo de la categoría es la práctica como investigación.

Lo que se observa es que con la ampliación de la enseñanza de grado en Servicio Social en los últimos años en Brasil, sobre todo de la enseñanza privada, ocurrió un aumento exponencial de la demanda por vacantes de prácticas, al mismo tiempo que había una carencia de profesionales para atender todo el contingente de estudiantes. Muchas instituciones de enseñanza, en especial instituciones privadas, pasaron a contratar asistentes sociales para ejecutar proyectos de extensión y/o de investigación con el objetivo de supervisar a estudiantes $y$, de esa forma, suplir la significativa demanda.

En algunos espacios colectivos, como en los Foros de Supervisión, se han relatado situaciones y experiencias diversas, algunas de las cuales presentan muchas controversias y divergencias con el proyecto de formación profesional y las normativas que regulan la supervisión de práctica en el Servicio Social.

La PNE afirma que la investigación no puede ser caracterizada como práctica, pues estos son momentos distintos, al mismo tiempo que se complementan en el proceso de formación y calificación profesional. El ejercicio profesional de asistentes sociales trae en sí la dimensión intervencionista y la dimensión investigativa como momentos dialécticamente vinculados.

A pesar de la naturaleza de indisociación entre intervención e investigación, cabe subrayar que la investigación, con sus particularidades, no puede ser configurada como práctica. La investigación es parte constitutiva y constituyente de todo el recorrido formativo, siendo competencia de las Instituciones de Enseñanza asegurarla de forma transversal en sus proyectos pedagógicos, lo que no significa reconocerla como práctica supervisada. Es necesario demarcar entre la práctica supervisada y la investigación, sus distinciones, particularidades, naturalezas $\mathrm{y}$, al mismo tiempo, vinculaciones $\mathrm{y}$ reciprocidades, en un todo en que se constituye la formación profesional. Esto no significa anular y/o negar la dimensión investigativa en el desarrollo y la realización de la práctica. Por el contrario, la práctica es un momento de aprehensión de la realidad social que se da por medio de síntesis y aproximaciones sucesivas de los componentes y contenidos curriculares que componen el proyecto de formación, en un movimiento que relaciona dialécticamente la realidad social y los fundamentos históricos, teóricos y metodológicos de la profesión y de la vida en sociedad. Es decir, en el nivel de la inmediatez y de la apariencia fenoménica, la realidad se presenta de manera caótica y abstracta a los/las estudiantes, ausente de mediaciones y contradicciones. Por eso, entender la realidad en su concreción, a la luz de fundamentos históricos, teóricos y sociales, posibilita la aproximación a la realidad, con sus mediaciones, contradicciones y determinaciones (Pontes, 2007).

Prospectiva. Revista de Trabajo Social e intervención social • No. $29 \bullet$ ene.-jun. $2020 \bullet$ pp. 39-56 e-ISSN: 2389-993X • Doi: 10.25100/prts.v0i29.7554

Universidad del Valle - Cali, Colombia 
En cuanto a la práctica en proyectos de extensión, la PNE prevé esa posibilidad siempre que se cumplan, reconozcan y caractericen como actividades que, de hecho, configuran acciones extensionistas y también deben estar contenidas y previstas en los Proyectos Pedagógicos de los cursos. La PNE también señala sobre posibles falacias en el sentido de desvirtuamiento de la extensión universitaria, lo que puede contribuir a atender intereses de grupos privados, con finalidad mercantil y lucrativa, reforzando acciones asistencialistas, filantrópicas, el trabajo voluntario, entre otras trampas contrarias a los valores y principios defendidos por el Servicio Social. Por eso, es fundamental que los proyectos de extensión estén sintonizados y sean coherentes con el proyecto ético-político profesional. La práctica en extensión no puede ser pensada como una solución burocrática para atender un requisito formal-institucional de las Instituciones de Enseñanza. La práctica debe ser comprendida en su totalidad, a partir de su dimensión pedagógica, en una formación que es dialécticamente procesal y dinámica, comprometida con valores emancipatorios.

Por lo tanto, se observa la necesidad de que la supervisión de práctica sea pauta constante en los foros de la categoría, para fortalecer espacios colectivos en la defensa de una formación profesional de calidad comprometida con los valores y principios del proyecto ético-político del Servicio Social.

\section{Conclusiones}

La construcción democrática y colectiva de la PNE en la gestión de la ABEPSS en el bienio 2009-2010, representó un marco histórico para el Servicio Social brasileño en el sentido de presentar principios orientadores del proceso de supervisión de prácticas que hasta entonces necesitaban ser delimitados y explicitados como forma de defender el proyecto de formación crítica construido a lo largo de los últimos años. Materializar los principios orientadores de la PNE en un escenario de precarización de las relaciones de trabajo y de una ofensiva reforma estructural que alcanza el sistema educativo brasileño significa resistir a las órdenes de la sociabilidad capitalista neoliberal.

La formación profesional se inserta en un movimiento contradictorio y complejo y, por eso, situar la supervisión de práctica en esta trama es entenderla como parte integrante de la totalidad social, que con sus múltiples determinaciones incide en la formación y en el cotidiano de trabajo de los/las asistentes sociales. Por eso, no se puede desconsiderar en ese análisis las tendencias de la política educativa brasileña, las transformaciones en el mundo del trabajo y la implementación del recetario neoliberal que impulsó procesos de privatización de los bienes, servicios y políticas públicas.

En vista de las tensiones existentes entre el proyecto ético-político del Servicio Social y las requisiciones del mercado de trabajo guiadas por la lógica capitalista neoliberal, se observa que el proyecto de formación profesional, sintonizado con una perspectiva crítica y emancipadora, defiende una formación de calidad, pública y laica, en que la supervisión de

Prospectiva. Revista de Trabajo Social e intervención social • No. $29 \bullet$ ene.jun. $2020 \bullet$ pp. 39-56 e-ISSN: 2389-993X • Doi: 10.25100/prts.v0i29.7554

Universidad del Valle - Cali, Colombia 
prácticas sea un componente central de ese proceso y tenga una dimensión pedagógica y formativa. La supervisión de prácticas (de campo y académica) es una asignación privada de asistentes sociales y la inserción de estudiantes en las prácticas permite su contacto y confrontación directa con la realidad social, con las demandas y necesidades de la población. Las prácticas supervisadas son también espacios privilegiados de desarrollo de habilidades y competencias (teóricas, metodológicas, éticas, políticas, técnicas, operativas e investigativas) de los estudiantes. Es decir, es el momento de síntesis entre formación y trabajo profesional, entre teoría y realidad social. La supervisión de práctica como componente pedagógico y formativo, permite la construcción colectiva de conocimientos, intercambios y experiencias entre los sujetos involucrados en este proceso, lo que resulta en la enseñanza-aprendizaje constante.

Por eso, implementar la PNE en el cotidiano de las Instituciones de Enseñanza superior y en los espacios laborales de asistentes sociales, asegurando sus principios y directrices, es defender el proyecto profesional crítico del Servicio Social, patrimonio histórico-colectivo de la categoría, con miras a la lucha por la educación pública, de calidad y laica. Es también defender mejores y dignas condiciones de trabajo para los/las profesionales, ante la reestructuración capitalista que desmonta gradualmente los derechos sociales, fragiliza y precariza los vínculos contractuales de trabajo.

\section{Referencias bibliográficas}

Associação Brasileira de Ensino e Pesquisa em Serviço Social. (1996a). Diretrizes Gerais para o curso de Serviço Social. Rio de Janeiro: ABEPSS.

Associação Brasileira de Ensino e Pesquisa em Serviço Social. (1996b). Proposta básica para o projeto de formação profissional. Revista Serviço Social e Sociedade, 50, 143-171.

Associação Brasileira de Ensino e Pesquisa em Serviço Social. (2009). Política Nacional de Estágio - PNE. Brasília: ABEPSS.

Baptista-Lewgoy, A. M. B. (2009). Supervisão de estágio em Serviço Social: desafios para a formação e o exercício profissional. São Paulo: Cortez.

Campanini, A. (2010). The challenges of Social Work education in Europe. Psychologica, 2(52), 687-700.

Chauí, M. (2003). A universidade pública sob nova perspectiva. Revista Brasileira de Educação, (24), 5-15.

Consejo Federal de Trabajo Social. (29 de septiembre de 2008). [Resolución No. 533 de 2008].

Consejo Federal de Trabajo Social. (7 de junio de 1993). Reglamento de la profesión de Asistente Social [Ley No. 8.662 de 1993]. DOU: 8.7.1993.

Iamamoto, M. (2007). Serviço Social em tempo de capital fetiche: capital financeiro, trabalho e questão social. São Paulo: Cortez.

Prospectiva. Revista de Trabajo Social e intervención social • No. $29 \bullet$ ene.-jun. 2020 • pp. 39-56 e-ISSN: 2389-993X • Doi: 10.25100/prts.v0i29.7554

Universidad del Valle - Cali, Colombia 
Marconsin, C. (2010). Documentação em Serviço Social: debatendo a concepção burocrática e rotineira. En Y. Guerra y V. Forti (Comps.), Serviço Social: temas, textos e contextos (pp. 65-76). Rio de Janeiro: Lumen Juris.

Netto, J.P. (1996). Transformações societárias e Serviço Social: notas para uma análise prospectiva da profissão no Brasil. Revista Serviço Social e Sociedade, (50), 87-132.

Oliveira, C. (2009). Estágio supervisionado curricular em Serviço Social: elementos para reflexão. Revista Temporalis, (17), 99-110.

Pontes, R. N. (2007). Mediação e Serviço social: um estudo preliminar sobre a categoria teórica e sua apropriação pelo Serviço Social. São Paulo: Cortez.

Presidencia de la República de Brasil. (19 de diciembre de 2005). [Decreto No. 5.622 de 2005]. DOU: 20.12.2005.

Presidencia de la República de Brasil. (24 de abril de 2007). [Decreto No. 6.096 de 2007]. DOU: 25.4.2007.

Presidencia de la República de Brasil. (25 de septiembre de 2008). Ley de Pasantías [Ley 11.788 de 2008]. DOU: 26.9.2008.

Presidencia de la República de Brasil. (26 de agosto de 2010). [Ley 12.317 de 2010]. DOU: 27.8.2010.

Puig-Cruells, C. (2015). La supervisión en la acción social: una oportunidad para el bienestar de los profesionales. Tarragona: URV.

Ramos, S. R. (2009). As Diretrizes Curriculares e a Política Nacional de Estágio: fundamentos, polêmicas e desafios. Revista Temporalis, (17), 21-37.

Vasconcelos, I. (2009). Dilemas e desafios do estágio curricular em Serviço Social: expressão dos (des) encontros entre a formação profissional e o mercado de trabalho. Revista Temporalis, (17), 61-82. 


\section{OTROS ARTÍCULOS DE PROSPECTIVA No. 29 DE 2020}

EDITORIAL

La Reconceptualización: una opción a la encrucijada del Trabajo Social en Colombia Víctor Mario Estrada-Ospina

\section{ARTÍCULOS}

Formación profesional y la práctica supervisada en el Servicio Social brasileño

Reginaldo Ghiraldelli

El rol docente del tutor y supervisor de prácticas de Trabajo Social: construcción de la reflexividad y el compromiso durante la formación a través de la supervisión

Carmina Puig-Cruells

Problematizando las prácticas preprofesionales en Trabajo Social. Desafios y perspectivas

Ruth Noemí Parola

Trabajo Social en contextos de formación política: la práctica transformadora

Angélica López-Yepes

Jackeline Cuenca-Echeverry

Investigación cuantitativa en una práctica universitaria de intervención en comunidad en el norte de México

Adán Cano-Aguilar
Interventores y comunidades vulnerables: una propuesta de formación para universitarios

Dulce Nayeli Cohetero-Hernández

Fidel González-Quiñones

Reagrupación familiar de menores en Aragón. Propuestas de acompañamiento en el proceso migratorio

Chabier Gimeno-Monterde

José David Gutiérrez-Sánchez

Aportaciones de la Educación en Derechos Humanos (EDH) y la Investigación Acción Participativa (IAP) en contextos de trabajo comunitario

Carla Cubillos-Vega

Condiciones para una NeoReconceptualización del Trabajo Social en Chile, Latinoamérica y el Caribe

Luis Vivero-Arriagada

Justicia sin daño: una apuesta por el fortalecimiento del acceso a la justicia Diego Fernando Acosta-Daza

\section{IN MEMORIAM}

Camilo Bautista Ochoa, un santandereano que se enamoró del Pacífico

Jesús Glay Mejía-Naranjo

\section{PROSPECTIVA}

Revista de Trabajo Social e Intervención Social 